\title{
Conjugate heat transfer for the unsteady compressible Navier-Stokes equations using a multi-block coupling
}

\author{
Jan Nordström and Jens Berg
}

\section{Linköping University Post Print}

N.B.: When citing this work, cite the original article.

Original Publication:

Jan Nordström and Jens Berg, Conjugate heat transfer for the unsteady compressible NavierStokes equations using a multi-block coupling, 2013, Computers \&amp; Fluids, (72), 20-29. http://dx.doi.org/10.1016/j.compfluid.2012.11.018

Copyright: Elsevier http://www.elsevier.com/

Postprint available at: Linköping University Electronic Press

http://urn.kb.se/resolve?urn=urn:nbn:se:liu:diva-86977 


\title{
Conjugate Heat Transfer for the Unsteady Compressible Navier-Stokes Equations Using a Multi-block Coupling
}

\author{
Jan Nordström ${ }^{\dagger *}$ \\ Department of Mathematics, Linköping University, SE-581 83 Linköping, Sweden \\ Jens Berg ${ }^{\dagger}$ \\ Department of Information Technology, Uppsala University, SE-751 05, Uppsala, Sweden
}

\begin{abstract}
This paper deals with conjugate heat transfer problems for the time-dependent compressible Navier-Stokes equations. One way to model conjugate heat transfer is to couple the Navier-Stokes equations in the fluid with the heat equation in the solid. This requires two different physics solvers. Another way is to let the Navier-Stokes equations govern the heat transfer in both the solid and in the fluid. This simplifies calculations since the same physics solver can be used everywhere.

We show by energy estimates that the continuous problem is well-posed when imposing continuity of temperature and heat fluxes by using a modified $L^{2}$-equivalent norm. The equations are discretized using finite difference on summation-by-parts form with boundary- and interface conditions imposed weakly by the simultaneous approximation term. It is proven that the scheme is energy stable in the modified norm for any order of accuracy.
\end{abstract}

\footnotetext{
*Corresponding author: Jan Nordström, E-mail: jan.nordstrom@liu.se

${ }^{\dagger}$ Parts of this work were completed while the authors visited the Centre for Turbulence Research at Stanford University
}

Preprint submitted to Elsevier

October 10, 2012 
We also show what is required for obtaining the same solution as when the unsteady compressible Navier-Stokes equations are coupled to the heat equation. The differences between the two coupling techniques are discussed theoretically as well as studied numerically, and it is shown that they are indeed small.

Keywords: Conjugate heat transfer, Navier-Stokes, compressible, unsteady, heat equation, finite difference, summation-by-parts, weak interface conditions, weak multi-block conditions, stability, high order accuracy

\section{Introduction}

Heat transfer is an important factor in many fluid dynamics applications. Flows are often confined within some material with heat transfer properties. Whenever there is a temperature difference between the fluid and the confining solid, heat will be transferred and change the flow properties in a non-trivial way. This interaction and heat exchange is referred to as the conjugate heat transfer problem $[1,2,3,4]$. Examples of application areas include cooling of turbine blades and nuclear reactors, atmospheric reentry of spacecrafts and gas propulsion micro thrusters for precise satellite navigation.

Conjugate heat transfer problems have been computed using a variety of methods. For stationary problems, methods include the finite volume method [5], the finite element method $[6,7]$ and the Semi-Implicit Method for Pressure-Linked Equations (SIMPLE) [8]. For unsteady problems, overlapping grids [3] and finite difference methods [1] have been used. The interface conditions have been imposed either strongly, weakly or by a mixture of both.

There are many ways in which conjugate heat transfer problems can be analyzed 
and computed. Giles [1] considered the simplified case of two coupled heat equations and performed a stability analysis which put restrictions on how to chose the interface conditions. Henshaw and Chand [3] performed numerical simulations of incompressible, temperature dependent fluids with the Boussinesq approximation coupled with the heat equation. The stability analysis was restricted to the case of two coupled heat equations. Stability and second order accuracy for the coupled model problem was proven, together with a numerical accuracy study of the full coupled problem showing second order accuracy, as expected. In [7] a steady, compressible fluid with heat transfer properties is considered and it is stated that accuracy is a key element in computational heat transfer. The authors develop an adaptive strategy with error estimators, showing at most second order accuracy.

When reviewing the literature on conjugate heat transfer problems, one can conclude that for incompressible problems, the heat transfer part is either modeled by the heat equation, or by using the incompressible Navier-Stokes equations also in the solid region. The latter strategy is possible since the energy equation in the incompressible Navier-Stokes equations decouples from the continuity- and momentum equations. In the compressible flow case, the situation is different and more complicated. Two major differences exist. Firstly, the energy equation does not decouple from the continuity- and momentum equations. Secondly, for compressible fluids, steady problems are mostly considered since the stability of the coupling becomes an issue.

The numerical methodology presented in this paper is based on a finite difference on Summation-By-Parts (SBP) form with the Simultaneous Approximation 
Term (SAT) for imposing the boundary and interface conditions weakly. The SBPSAT method has been used for a variety of problems and has proven to be robust $[9,10,11,12,13,14,15]$. The SBP finite difference operators were originally constructed by Kreiss and Schearer [16] with the purpose of constructing an energy stable finite difference method [17]. Together with the weak imposition of boundary [18] and interface [19] conditions, the SBP-SAT provides a method for constructing energy stable schemes for well-posed initial-boundary value problems [20]. There are SBP operators based on diagonal norms for the first [21] and second [22, 23] derivative accurate of order 2, 3, 4 and 5, and the stability analysis we will present is independent of the order of accuracy.

From an implementational point of view, coupling the compressible Navier-Stokes equations to the heat equation is complicated as different solvers are required in the fluid and solid domains. With two different solvers, two different codes, are required and data has to be transferred between them by using possibly a third code [24].

A less complicated method would be to only use the Navier-Stokes equations everywhere and modify an already existing multi-block coupling [12] such that heat is transferred between the fluid and solid domains. In the blocks marked as solids, it is possible to construct initial and boundary conditions such that the velocities and density gradients are small. The difference between the energy component of the compressible Navier-Stokes equations and the heat equation should then also be small.

We will show how to scale and choose the coefficients of the energy part of the Navier-Stokes equations, such that it is as similar to the heat equation as possible. 
Numerical simulations of heat transfer in solids are performed to show the similarities, and differences, of the temperature distributions obtained by the Navier-Stokes equations and the heat equation. We will not overwrite, or strongly force, the velocities in the Navier-Stokes equations to zero in each time integration stage since that would ruin the stability of the numerical method that we use. Instead, the velocities will be enforced weakly at the boundaries and interfaces only.

In the previous literature, a mathematical investigation of the interface conditions in terms of well-posedness of the continuous equations, stability of the resulting numerical scheme and high order accuracy has not been performed to our knowledge. We shall in this paper hence focus on the mathematical treatment of the fluid-solid interface rather than computing physically relevant scenarios.

\section{The compressible Navier-Stokes equations}

The two-dimensional compressible Navier-Stokes equations in dimensional, conservative form are

$$
q_{t}+F_{x}+G_{y}=0
$$

where the conserved variables, $q=[\rho, \rho u, \rho v, e]^{T}$, are the density, $\mathrm{x}$ - and $\mathrm{y}$-directional momentum and energy, respectively. The energy is given by

$$
e=c_{V} \rho T+\frac{1}{2} \rho\left(u^{2}+v^{2}\right)
$$

where $c_{V}$ is the specific heat capacity under constant volume and $T$ is the temperature. Furthermore, we have $F=F^{I}-F^{V}$ and $G=G^{I}-G^{V}$, where the superscript 
$I$ denotes the inviscid part of the flux and $V$ the viscous part. The components of the flux vectors are given by

$$
\begin{aligned}
F^{I} & =\left[\rho u, p+\rho u^{2}, \rho u v, u(p+e)\right]^{T}, \\
G^{I} & =\left[\rho v, \rho u v, p+\rho v^{2}, v(p+e)\right]^{T}, \\
F^{V} & =\left[0, \tau_{x x}, \tau_{x y}, u \tau_{x x}+v \tau_{x y}+\kappa T_{x}\right]^{T}, \\
G^{V} & =\left[0, \tau_{x y}, \tau_{y y}, u \tau_{y x}+v \tau_{y y}+\kappa T_{y}\right]^{T},
\end{aligned}
$$

where we have the pressure $p$ and the thermal conductivity coefficient $\kappa$. The stress tensor is given by

$\tau_{x x}=2 \mu \frac{\partial u}{\partial x}+\lambda\left(\frac{\partial u}{\partial x}+\frac{\partial v}{\partial y}\right), \tau_{y y}=2 \mu \frac{\partial v}{\partial y}+\lambda\left(\frac{\partial u}{\partial x}+\frac{\partial v}{\partial y}\right), \tau_{x y}=\tau_{y x}=\mu\left(\frac{\partial u}{\partial y}+\frac{\partial v}{\partial x}\right)$,

where $\mu$ and $\lambda$ are the dynamic and second viscosity, respectively. To close the system we need to include an equation of state, for example the ideal gas law

$$
p=\rho R T .
$$

Here $R=c_{P}-c_{V}$ is the specific gas constant and $c_{P}$ the specific heat capacity under constant pressure. Both $c_{P}$ and $c_{V}$ are considered constants in this paper.

Since the aim is to model heat transfer in a solid using the Navier-Stokes equations, we study the equations with vanishing velocities. If we let $u=v=0$, all the convective terms and viscous stresses are zero and by using (2) and (5), equation (1) 
reduces to

$$
\begin{aligned}
\rho_{t} & =0 \\
p_{x} & =0 \\
p_{y} & =0 \\
T_{t} & =\frac{1}{c_{V} \rho}\left(\left(\kappa T_{x}\right)_{x}+\left(\kappa T_{y}\right)_{y}\right) .
\end{aligned}
$$

The last equation is similar, but not identical, to the variable coefficient heat equation.

For ease of comparison with the heat equation we transform to non-dimensional form as follows (note the slight abuse of notation since we let the dimensional and non-dimensional variables have the same notation. Hereafter, all quantities are nondimensional):

$$
\begin{aligned}
& u=\frac{u^{*}}{c_{\infty}^{*}}, \\
& v=\frac{v^{*}}{c_{\infty}^{*}}, \\
& \rho=\frac{\rho^{*}}{\rho_{\infty}^{*}}, \\
& T=\frac{T^{*}}{T_{\infty}^{*}}, \\
& p=\frac{p^{*}}{\rho_{\infty}^{*}\left(c_{\infty}^{*}\right)^{2}}, \\
& e=\frac{e^{*}}{\rho_{\infty}^{*}\left(c_{\infty}^{*}\right)^{2}}, \\
& \lambda=\frac{\lambda^{*}}{\mu_{\infty}^{*}}, \\
& \mu=\frac{\mu^{*}}{\mu_{\infty}^{*}}, \\
& c_{P}=\frac{c_{P}^{*}}{c_{P \infty}^{*}}, \\
& c_{V}=\frac{c_{V}^{*}}{c_{P \infty}^{*}}, \\
& R=\frac{R^{*}}{c_{P \infty}^{*}}, \\
& \kappa=\frac{\kappa^{*}}{\kappa_{\infty}^{*}}, \\
& x=\frac{x^{*}}{L_{\infty}^{*}}, \\
& y=\frac{y^{*}}{L_{\infty}^{*}}, \\
& t=\frac{c_{\infty}^{*}}{L_{\infty}^{*}} t^{*},
\end{aligned}
$$

where the $*$-superscript denotes a dimensional variable and the $\infty$-subscript the reference value. $L_{\infty}^{*}$ is a characteristic length scale and $c_{\infty}^{*}$ is the reference speed of 
sound. The equation of state (5) becomes in non-dimensional form

$$
\gamma p=\rho T .
$$

and the energy equation can be written as

$$
e=\frac{p}{\gamma-1}+\frac{1}{2} \rho\left(u^{2}+v^{2}\right) .
$$

By using (7)-(10), the last equation in (6) becomes

$$
T_{t}=\frac{1}{P e_{c}} \frac{1}{c_{V} \rho}\left(\left(\kappa T_{x}\right)_{x}+\left(\kappa T_{y}\right)_{y}\right)
$$

where

$$
P e_{c}=\frac{c_{\infty}^{*} L_{\infty}^{*}}{\alpha_{\infty}^{*}}, \quad \alpha_{\infty}^{*}=\frac{\kappa_{\infty}^{*}}{\rho_{\infty}^{*} c_{P \infty}^{*}}
$$

are the Péclet number based on the reference speed of sound and the thermal diffusivity, respectively.

\section{Similarity conditions}

Since the fluid is compressible, the density in (6) is non-constant and the energy component in the Navier-Stokes equations will differ from the constant coefficient heat equation. We can however quantify in which way the equations differ and which

terms that have to be minimized in order for the two equations to be as similar as 
possible. The heat equation, non-dimensionalized using (7)-(10), can be written as

$$
\tilde{T}_{t}=\frac{1}{P e_{c}} \frac{1}{c_{s} \rho_{s}}\left(\left(\kappa_{s} \tilde{T}_{x}\right)_{x}+\left(\kappa_{s} \tilde{T}_{y}\right)_{y}\right)
$$

where $P e_{c}$ is defined in (14) and $c_{s}, \rho_{s}, \kappa_{s}$ are the specific heat capacity, density and thermal conductivity of the solid, respectively. In this case, all coefficients are constant but rewritten in a form which resembles (13).

In order to compare (13) and (15), we define $\beta=P e_{c} \rho c_{V}, \beta_{s}=P e_{c} \rho_{s} c_{s}$ and rewrite (13) and (15) as

$$
\begin{aligned}
& \beta T_{t}=\left(\kappa T_{x}\right)_{x}+\left(\kappa T_{y}\right)_{y}, \\
& \beta \tilde{T}_{t}=\frac{\beta}{\beta_{s}}\left(\left(\kappa_{s} \tilde{T}_{x}\right)_{x}+\left(\kappa_{s} \tilde{T}_{y}\right)_{y}\right) .
\end{aligned}
$$

Note that $\beta_{s}$ is constant for the solid. Furthermore, since $\beta>0$ and (6) yields $\frac{\partial \beta}{\partial t}=0$, we can estimate the difference $T-\tilde{T}$ in the $\beta$-norm defined by

$$
\|T-\tilde{T}\|_{\beta}^{2}=\int_{\Omega}(T-\tilde{T})^{2} \beta d \Omega
$$

where $\Omega$ is the computational domain. By subtracting (17) from (16), multiplying 
with $T-\tilde{T}$ and integrating over $\Omega$ we obtain

$$
\begin{aligned}
\frac{1}{2} \frac{d}{d t}\|T-\tilde{T}\|_{\beta}^{2} & =-\int_{\Omega}\left(\kappa \nabla T \cdot \nabla T+\frac{\kappa_{s}}{\beta_{s}} \beta \nabla \tilde{T} \cdot \nabla \tilde{T}\right) \\
& +\oint_{\partial \Omega}(T-\tilde{T})\left(\kappa \nabla T-\frac{\kappa_{s}}{\beta_{s}} \beta \nabla \tilde{T}\right) \cdot n d s \\
& +\int_{\Omega} \frac{\kappa_{s}}{\beta_{s}}(T-\tilde{T}) \nabla \beta \cdot \nabla \tilde{T} d \Omega+\int_{\Omega}\left(\kappa+\frac{\kappa_{s}}{\beta_{s}} \beta\right) \nabla T \cdot \nabla \tilde{T} d \Omega .
\end{aligned}
$$

In order to obtain as similar temperature distributions from the heat equation and Navier-Stokes equation as possible, the right-hand-side of (19) has to be less than or

equal to zero. Note that we specify the same boundary data for $T$ and $\tilde{T}$, in which case the boundary integral is zero. By further assuming that $\nabla \beta=0$ we can rewrite (19) as the quadratic form

$$
\frac{d}{d t}\|T-\tilde{T}\|_{\beta}^{2}=-\int_{\Omega}\left[\begin{array}{c}
\nabla T \\
\nabla \tilde{T}
\end{array}\right]^{T}\left[\begin{array}{cc}
2 \kappa & -\left(\kappa+\frac{\kappa_{s}}{\beta_{s}} \beta\right) \\
-\left(\kappa+\frac{\kappa_{s}}{\beta_{s}} \beta\right) & 2 \frac{\kappa_{s}}{\beta_{s}} \beta
\end{array}\right]\left[\begin{array}{c}
\nabla T \\
\nabla \tilde{T}
\end{array}\right] .
$$

By computing the eigenvalues of the matrix in (20) and requiring that they be nonnegative, we can conclude that we need $\kappa-\frac{\kappa_{s}}{\beta_{s}} \beta=0$. Thus, if the relations

$$
\frac{\kappa}{\beta}-\frac{\kappa_{s}}{\beta_{s}}=0, \quad \nabla \beta=0
$$

hold, then

$$
\frac{d}{d t}\|T-\tilde{T}\|_{\beta}^{2} \leq 0
$$


and the Navier-Stokes equations and the heat equation produces the exact same solution for the temperature if given identical initial data.

Remark 1. The heat equation and energy component in the Navier-Stokes equations produces exactly the same results only if the relations in (21) hold. In a numerical simulation, the initial, and boundary, data are chosen such that (21) holds exactly to begin with. Because of the weak imposition of the boundary and interface conditions, the relations will no longer hold as time passes. Small variations in the velocities at the boundaries and interfaces will produce small variations in the density which propagate into the domain. These deviations are however very small and the effects are studied in later sections.

\section{SBP-SAT discretization}

In the basic formulation, the first derivative is approximated by an operator on SBP form

$$
u_{x} \approx D v=P^{-1} Q v,
$$

where $v$ is the discrete grid function approximating $u$. The matrix $P$ is symmetric,

positive definite and defines a discrete norm by $\|v\|^{2}=v^{T} P v$. In this paper, we consider diagonal norms only. The matrix $Q$ is almost skew-symmetric and satisfies the SBP property $Q+Q^{T}=\operatorname{diag}[-1,0, \ldots, 0,1]$. There are SBP operators based on diagonal norms with 2nd, 3rd, 4th and 5th order accuracy, and the stability analysis does not depend on the order of the operators $[21,25]$. The second derivative is 
approximated either using the first derivative twice, i.e.

$$
u_{x x} \approx D^{2} v=\left(P^{-1} Q\right)^{2} v
$$

or a compact formulation with minimal bandwidth $[22,23]$. In the conservative formulation of the Navier-Stokes equations, the second derivative operator is not used.

In order to extend the operators to higher dimensions, it is convenient to introduce the Kronecker product. For arbitrary matrices $A \in \mathbb{R}^{m \times n}$ and $B \in \mathbb{R}^{p \times q}$, the Kronecker product is defined as

$$
A \otimes B=\left[\begin{array}{ccc}
a_{1,1} B & \ldots & a_{1, m} B \\
\vdots & \ddots & \vdots \\
a_{n, 1} B & \ldots & a_{m, n} B
\end{array}\right]
$$

The Kronecker product is bilinear, associative and obeys the mixed product property

$$
(A \otimes B)(C \otimes D)=(A C \otimes B D)
$$

if the usual matrix products are defined. For inversion and transposing we have

$$
(A \otimes B)^{-1, T}=A^{-1, T} \otimes B^{-1, T}
$$

if the usual matrix inverse is defined. The Kronecker product is not commutative in 
general, but for square matrices $A$ and $B$ there is a permutation matrix $R$ such that

$$
A \otimes B=R^{T}(B \otimes A) R .
$$

Let $P_{x, y}, Q_{x, y}$ and $D_{x, y}$ denote the difference operators in the coordinate direction indicated by the subscript. The extension to multiple dimensions is done by using the Kronecker product as follows:

$$
\begin{array}{ll}
\bar{P}_{x}=P_{x} \otimes I_{y}, & \bar{Q}_{x}=Q_{x} \otimes I_{y}, \\
\bar{P}_{y}=I_{x} \otimes P_{y}, & \bar{Q}_{y}=I_{x} \otimes Q_{y}, \\
\bar{D}_{x}=D_{x} \otimes I_{y}, & \bar{D}_{y}=I_{x} \otimes D_{y} .
\end{array}
$$

Due to the mixed product property (26), the operators commute in different coordinate directions and hence differentiation can be performed in each coordinate direction independently. The norm is defined by

$$
\|u\|^{2}=u^{T} \bar{P} u
$$

where $\bar{P}=\bar{P}_{x} \bar{P}_{y}=P_{x} \otimes P_{y}$.

\section{Temperature coupling of the Navier-Stokes equations}

The compressible Navier-Stokes equations in two space dimensions requires three boundary conditions at a solid wall [20]. Since we are aiming for modelling heat transfer in a solid using (1), both the tangential and normal velocities are zero. The 
third condition is used to couple the temperature in the fluid and solid domain.

We consider the Navier-Stokes equations in the two domains $\Omega_{1}=[0,1] \times[0,1]$ and $\Omega_{2}=[0,1] \times[-1,0]$ with an interface at $y=0$. Denote the solution in $\Omega_{1}$ by $q=[\rho, \rho u, \rho v, e]$ and in $\Omega_{2}$ by $\tilde{q}=[\tilde{\rho}, \tilde{\rho} \tilde{u}, \tilde{\rho} \tilde{v}, \tilde{e}]$.

The interface will be considered as a solid wall and hence we impose no-slip interface conditions for the velocities

$$
\begin{aligned}
& u=0, \quad v=0, \\
& \tilde{u}=0, \quad \tilde{v}=0 .
\end{aligned}
$$

More general interface conditions can be imposed by considering Robin conditions as described in [26].

To couple the temperature of the two equations we will use continuity of temperature and heat fluxes,

$$
T=\tilde{T}, \quad \kappa_{1} T_{y}=\kappa_{2} \tilde{T}_{y}
$$

For the purpose of analysis, we consider the linearized, frozen coefficient and symmetric Navier-Stokes equations

$$
\begin{aligned}
& w_{t}+\left(A_{1} w\right)_{x}+\left(A_{2} w\right)_{y}=\varepsilon\left(\left(A_{11} w_{x}+A_{12} w_{y}\right)_{y}+\left(A_{21} w_{x}+A_{22} w_{y}\right)_{y}\right), \\
& \tilde{w}_{t}+\left(B_{1} \tilde{w}\right)_{x}+\left(B_{2} \tilde{w}\right)_{y}=\varepsilon\left(\left(B_{11} \tilde{w}_{x}+B_{12} \tilde{w}_{y}\right)_{y}+\left(B_{21} \tilde{w}_{x}+B_{22} \tilde{w}_{y}\right)_{y}\right),
\end{aligned}
$$

where $\epsilon=\frac{M a}{R e}, R e$ is the Reynolds number and $M a$ is the Mach number. The 
coefficient matrices can be found in [20, 27]. The symmetrized variables are

$$
w=\left[\frac{\bar{c}}{\sqrt{\gamma} \bar{\rho}} \rho, u, v, \frac{1}{\bar{c} \sqrt{\gamma(\gamma-1)}} T\right]^{T}
$$

where an overbar denotes the constant state which we have linearized around. More details can be found in $[28,20,27]$. This procedure is motivated by the principle of linearization and localization [29]. Note that the linerarization around $u=v=0$, and hence $\bar{u}=\bar{v}=0$, is exact at the interface due to the interface conditions. The well-posedness of (33) with the conditions (31) and (32) are shown in

Proposition 1. The coupled compressible Navier-Stokes equations are well-posed using the interface conditions (31) and (32).

Proof. The energy estimates of $w$ and $\tilde{w}$ will be derived in the $L^{2}$-equivalent norms

$$
\|w\|_{H_{1}}^{2}=\int_{\Omega_{1}} w^{T} H_{1} w d \Omega, \quad\|\tilde{w}\|_{H_{2}}^{2}=\int_{\Omega_{2}} \tilde{w}^{T} H_{2} \tilde{w} d \Omega
$$

where

$$
H_{1,2}=\operatorname{diag}\left[1,1,1, \delta_{1,2}\right], \quad \delta_{1,2}>0
$$

are to be determined. We apply the energy method and consider only the terms at the interface $y=0$. We get by using the conditions (31) that

$$
\frac{d}{d t}\left(\|w\|_{H_{1}}^{2}+\|\tilde{w}\|_{H_{2}}^{2}\right) \leq-2 \varepsilon \int_{0}^{1}\left(\frac{\delta_{1} \bar{\mu}_{1}}{\bar{\rho}_{1} \bar{c}_{1}^{2}\left(\gamma_{1}-1\right) P r_{1}} T T_{y}-\frac{\delta_{2} \bar{\mu}_{2}}{\bar{\rho}_{2} \bar{c}_{2}^{2}\left(\gamma_{2}-1\right) P r_{2}} \tilde{T} \tilde{T} y\right) d x
$$

where the bar denotes the state around which we have linearized and the subscript 
1 or 2 refer to values from the corresponding subdomain $\Omega_{1}$ or $\Omega_{2}$. By requiring continuity of temperature $(T=\tilde{T})$ equation (37) reduces to

$$
\frac{d}{d t}\left(\|w\|_{H_{1}}^{2}+\|\tilde{w}\|_{H_{2}}^{2}\right) \leq-2 \varepsilon \int_{0}^{1} T\left(\frac{\delta_{1} \bar{\kappa}_{1}}{\bar{\rho}_{1} \bar{c}_{1}^{2}\left(\gamma_{1}-1\right) c_{P_{1}}} T_{y}-\frac{\delta_{2} \bar{\kappa}_{2}}{\bar{\rho}_{2} \bar{c}_{2}^{2}\left(\gamma_{2}-1\right) c_{P_{2}}} \tilde{T}_{y}\right) d x .
$$

In order to obtain an energy estimate by using continuity of the heat fluxes, we need to choose the weights

$$
\delta_{1}=\bar{\rho}_{1} \bar{c}_{1}^{2}\left(\gamma_{1}-1\right) c_{P_{1}}, \quad \delta_{2}=\bar{\rho}_{2} \bar{c}_{2}^{2}\left(\gamma_{2}-1\right) c_{P_{2}}
$$

since then

$$
\frac{d}{d t}\left(\|w\|_{H_{1}}^{2}+\|\tilde{w}\|_{H_{2}}^{2}\right) \leq-2 \varepsilon \int_{0}^{1} T\left(\bar{\kappa}_{1} T_{y}-\bar{\kappa}_{2} \tilde{T}_{y}\right) d x=0 .
$$

Hence the interface conditions (32) gives an energy estimate and no unbounded energy growth can occur.

Remark 2. The physical interface conditions (32) requires an estimate in a different norm than the standard $L^{2}$-norm. The norm defined by the (positive) weights in (39) is, however, only a scaling of the $L^{2}$-norm and they are hence equivalent. From a mathematical point of view, any interface condition which give positive weights will result in a well-posed coupling. 


\subsection{The discrete problem and stability}

In [12], a stable and conservative multi-block coupling of the Navier-Stokes equations was developed. The coupling was done by considering continuity of all quantities and of the fluxes with the purpose of being able to handle different coordinate transforms in different blocks. In our case, the velocities are uncoupled and the equations are coupled only by continuity of temperature and heat fluxes. This enable us to compute conjugate heat problems by modifying the interface conditions for the multi-block coupling.

We consider again the formulation (33) and discretize using SBP-SAT for imposing the interface conditions (31) and (32) weakly. We let for simplicity the subdomains be discretized by equally many uniformly distributed gridpoints which allow us to use the same difference operators in both subdomains. We stress that the subdomains can have different discretizations $[12,30]$, this assumption merely simplifies the notation and avoids the use of too many subscripts.

We discretize (33) using the SBP-SAT technique as

$$
\begin{aligned}
& \mathbf{w}_{t}+\hat{D}_{x} \mathbf{F}+\hat{D}_{y} \mathbf{G}=\mathbb{S}, \\
& \tilde{\mathbf{w}}_{t}+\hat{D}_{x} \tilde{\mathbf{F}}+\hat{D}_{y} \tilde{\mathbf{G}}=\tilde{\mathbb{S}},
\end{aligned}
$$


where the discrete fluxes are given by

$$
\begin{array}{r}
\mathbf{F}=\hat{A}_{1} \mathbf{w}-\varepsilon\left(\hat{A}_{11} \hat{D}_{x} \mathbf{w}+\hat{A}_{12} \hat{D}_{y} \mathbf{w}\right), \\
\mathbf{G}=\hat{A}_{2} \mathbf{w}-\varepsilon\left(\hat{A}_{21} \hat{D}_{x} \mathbf{w}+\hat{A}_{22} \hat{D}_{y} \mathbf{w}\right), \\
\tilde{\mathbf{F}}=\hat{B}_{1} \tilde{\mathbf{w}}-\varepsilon\left(\hat{B}_{11} \hat{D}_{x} \tilde{\mathbf{w}}+\hat{B}_{12} \hat{D}_{y} \tilde{\mathbf{w}}\right), \\
\tilde{\mathbf{G}}=\hat{B}_{2} \tilde{\mathbf{w}}-\varepsilon\left(\hat{B}_{21} \hat{D}_{x} \tilde{\mathbf{w}}+\hat{B}_{22} \tilde{D}_{y} \tilde{\mathbf{w}}\right) .
\end{array}
$$

The hat notation denotes that the matrix has been extended to the entire system as

$$
\begin{aligned}
& \hat{D}_{x}=D_{x} \otimes I_{y} \otimes I_{4}, \quad \hat{D}_{y}=I_{x} \otimes D_{y} \otimes I_{4}, \\
& \hat{A}_{\xi}=I_{x} \otimes I_{y} \otimes A_{\xi}, \quad \hat{B}_{\xi}=I_{x} \otimes I_{y} \otimes B_{\xi},
\end{aligned}
$$

where $\xi$ is a generic index ranging over the indicies which occur in (42).

The SATs imposing the interface conditions (31) and (32) can be written as

$$
\begin{aligned}
\mathbb{S} & =\hat{P}_{y}^{-1} \hat{E}_{x, y_{N}} \hat{\Sigma}_{1}\left(\mathbf{w}-g^{I}\right)+\varepsilon \sigma_{2} \hat{P}_{y}^{-1} \hat{E}_{x, y_{N}}\left(\hat{H}_{2} \mathbf{w}-g_{1}\right) \\
& +\varepsilon \sigma_{3} \hat{P}_{y}^{-1} \hat{E}_{x, y_{N}}\left(\hat{H}_{3} \mathbf{w}-g_{2}\right)+\varepsilon \hat{P}_{y}^{-1} \hat{E}_{x, y_{N}} \hat{\Theta}_{1}\left(\hat{H}_{3} \hat{D}_{x} \mathbf{w}-\frac{\partial g_{2}}{\partial x}\right) \\
& +\varepsilon \sigma_{4} \hat{P}_{y}^{-1} \hat{E}_{x, y_{N}}\left(\hat{I}_{1}^{T} \mathbf{w}-\hat{I}_{2}^{T} \tilde{\mathbf{w}}\right) \\
& +\varepsilon \sigma_{5} \hat{P}_{y}^{-1} \hat{D}_{y}^{T} \hat{E}_{x, y_{N}}\left(\hat{I}_{1}^{T} \mathbf{w}-\hat{I}_{2}^{T} \tilde{\mathbf{w}}\right) \\
& +\varepsilon \sigma_{6} \hat{P}_{y}^{-1} \hat{E}_{x, y_{N}}\left(\bar{\kappa}_{1} \hat{I}_{1}^{T} \hat{D}_{y} \mathbf{w}-\bar{\kappa}_{2} \hat{I}_{2}^{T} \hat{D}_{y} \tilde{\mathbf{w}}\right)
\end{aligned}
$$


and

$$
\begin{aligned}
\widetilde{\mathbb{S}} & =\hat{P}_{y}^{-1} \hat{E}_{x, y_{0}} \hat{\Sigma}_{2}\left(\tilde{\mathbf{w}}-\tilde{g}^{I}\right)+\varepsilon \tilde{\sigma}_{2} \hat{P}_{y}^{-1} \hat{E}_{x, y_{0}}\left(\hat{H}_{2} \tilde{\mathbf{w}}-\tilde{g}_{1}\right) \\
& +\varepsilon \tilde{\sigma}_{3} \hat{P}_{y}^{-1} \hat{E}_{x, y_{0}}\left(\hat{H}_{3} \tilde{\mathbf{w}}-\tilde{g}_{2}\right)+\varepsilon \hat{P}_{y}^{-1} \hat{E}_{x, y_{0}} \hat{\Theta}_{2}\left(\hat{H}_{3} \hat{D}_{x} \tilde{\mathbf{w}}-\frac{\partial \tilde{g}_{2}}{\partial x}\right) \\
& +\varepsilon \tilde{\sigma}_{4} \hat{P}_{y}^{-1} \hat{E}_{x, y_{0}}\left(\hat{I}_{2}^{T} \tilde{\mathbf{w}}-\hat{I}_{1}^{T} \mathbf{w}\right) \\
& +\varepsilon \tilde{\sigma}_{5} \hat{P}_{y}^{-1} \hat{D}_{y}^{T} \hat{E}_{x, y_{0}}\left(\hat{I}_{2}^{T} \tilde{\mathbf{w}}-\hat{I}_{1}^{T} \mathbf{w}\right) \\
& +\varepsilon \tilde{\sigma}_{6} \hat{P}_{y}^{-1} \hat{E}_{x, y_{0}}\left(\bar{\kappa}_{2} \hat{I}_{2}^{T} \hat{D}_{y} \tilde{\mathbf{w}}-\bar{\kappa}_{1} \hat{I}_{1}^{T} \hat{D}_{y} \mathbf{w}\right) .
\end{aligned}
$$

Here $\hat{P}=\bar{P} \otimes I_{4}, \hat{E}_{x, y_{0}}=\bar{E}_{x, y_{0}} \otimes I_{4}, \hat{H}_{j}=I_{x} \otimes I_{y} \otimes H_{j}$ and $H_{j}$ is a $4 \times 4$ matrix with the only non-zero element 1 at the $(j, j)$ th position on the diagonal and the operators $\hat{I}_{1,2}$ selects the interface elements. The penalty matrices $\hat{\Sigma}_{1,2}=I_{x} \otimes I_{y} \otimes \Sigma_{1,2}$, $\hat{\Theta}_{1,2}=I_{x} \otimes I_{y} \otimes \Theta_{1,2}$, and the penalty coefficients $\sigma_{2, \ldots, 6}$ and $\tilde{\sigma}_{2, \ldots, 6}$ has to be determined such that the scheme is stable.

Remark 3. The terms which involve $\hat{\Theta}_{1,2}$ originate from the fact that the boundary condition $v=0$ implies that $v_{x}=0$, which is used to obtain an energy estimate in the continuous case. The terms hence represent the artificial boundary condition $v_{x}=0$ which is needed to obtain an energy estimate in the discrete case.

Remember that in the energy estimates for the continuous coupling, a nonstandard $L^{2}$-equivalent norm was used. The same modification to the norms has to be done in the discrete case. Thus, the discrete energy estimates will be derived in the norms

$$
\|\mathbf{w}\|_{\hat{J}_{1}}^{2}=\mathbf{w}^{T} \hat{P} \hat{J}_{1} \mathbf{w}, \quad\|\tilde{\mathbf{w}}\|_{\hat{J}_{2}}^{2}=\tilde{\mathbf{w}}^{T} \hat{P} \hat{J}_{2} \tilde{\mathbf{w}}
$$


where,

$$
\hat{J}_{1}=I_{x} \otimes I_{y} \otimes H_{1}, \quad \hat{J}_{2}=I_{x} \otimes I_{y} \otimes H_{2},
$$

and the matrices $H_{1,2}$ are defined in (36) with the weights given in (39). Note that $\hat{P} \hat{J}_{1,2}=\hat{J}_{1,2} \hat{P}$.

By applying the energy method to (41) and adding up we get

$$
\frac{d}{d t}\|\mathbf{w}\|_{\hat{J}_{1}}^{2}+\frac{d}{d t}\|\tilde{\mathbf{w}}\|_{\hat{J}_{2}}^{2}+D I=I T
$$

where the dissipation term, $D I$, is given by

$$
\begin{aligned}
D I & =2 \varepsilon\left[\begin{array}{c}
\hat{D}_{x} \mathbf{w} \\
\hat{D}_{y} \mathbf{w}
\end{array}\right]^{T}\left[\begin{array}{cc}
\hat{P} \hat{J}_{1} & 0 \\
0 & \hat{P} \hat{J}_{1}
\end{array}\right]\left[\begin{array}{cc}
\hat{A}_{11} & \hat{A}_{12} \\
\hat{A}_{21} & \hat{A}_{22}
\end{array}\right]\left[\begin{array}{c}
\hat{D}_{x} \mathbf{w} \\
\hat{D}_{y} \mathbf{w}
\end{array}\right] \\
& +2 \varepsilon\left[\begin{array}{c}
\hat{D}_{x} \tilde{\mathbf{w}} \\
\hat{D}_{y} \tilde{\mathbf{w}}
\end{array}\right]^{T}\left[\begin{array}{cc}
\hat{P} \hat{J}_{2} & 0 \\
0 & \hat{P} \hat{J}_{2}
\end{array}\right]\left[\begin{array}{cc}
\hat{B}_{11} & \hat{B}_{12} \\
\hat{B}_{21} & \hat{B}_{22}
\end{array}\right]\left[\begin{array}{c}
\hat{D}_{x} \tilde{\mathbf{w}} \\
\hat{D}_{y} \tilde{\mathbf{w}}
\end{array}\right] .
\end{aligned}
$$

The interface terms can be split into three parts as $I T=I T_{1}+I T_{2}+I T_{3}$ where $I T_{1}$ are the inviscid terms, $I T_{2}$ the velocity terms and $I T_{3}$ the coupling terms related to the temperature.

In [26] it is shown how to choose $\Sigma_{1,2}, \Theta_{1,2}, \sigma_{2,3}$ and $\tilde{\sigma}_{2,3}$, with small modifications, such that the inviscid and velocity terms are bounded. Here we focus on the coupling terms. With appropriate choices of $\Sigma_{1,2}, \Theta_{1,2}, \sigma_{2,3}$ and $\tilde{\sigma}_{2,3}$ as described in [26] we get

$$
\frac{d}{d t}\|\mathbf{w}\|_{H_{1}}^{2}+\frac{d}{d t}\|\tilde{\mathbf{w}}\|_{H_{2}}^{2}+D I \leq I T_{3}
$$


where $I T_{3}$ can be written as the quadratic form

$$
I T_{3}=-\varepsilon(R \xi)^{T}\left(\mathbf{P}_{x} \otimes M\right) R \xi
$$

To obtain (51), we have used the permutation similarity property of the Kronecker product, $R$ is a permutation matrix and $\xi=\left[\mathbf{T}_{i}, \tilde{\mathbf{T}}_{i},\left(D_{y} \mathbf{T}\right)_{i},\left(D_{y} \tilde{\mathbf{T}}\right)_{i}\right]^{T}$ where the subscript $i$ denotes the values at the interface. Note that we do not need the specific form of $R$, it is sufficient to know that such a matrix exists. Furthermore, we have

$$
\mathbf{P}_{x}=\operatorname{diag}\left[\delta_{1} P_{x}, \delta_{2} P_{x}, \delta_{1} P_{x}, \delta_{2} P_{x}\right]
$$

with $\delta_{1,2}$ from $(39)$, and

$$
M=\left[\begin{array}{cccc}
-2 \sigma_{4} & \sigma_{4}+\tilde{\sigma}_{4} & \bar{\kappa}_{1} \gamma_{1}-\sigma_{5}-\bar{\kappa}_{1} \sigma_{6} & \bar{\kappa}_{2} \sigma_{6}+\tilde{\sigma}_{5} \\
\sigma_{4}+\tilde{\sigma}_{4} & -2 \tilde{\sigma}_{4} & \sigma_{5}+\bar{\kappa}_{1} \tilde{\sigma}_{6} & -\bar{\kappa}_{2} \gamma_{1}-\tilde{\sigma}_{5}-\bar{\kappa}_{2} \tilde{\sigma}_{6} \\
\bar{\kappa}_{1} \gamma_{1}-\sigma_{5}-\bar{\kappa}_{1} \sigma_{6} & \sigma_{5}+\bar{\kappa}_{1} \tilde{\sigma}_{6} & 0 & 0 \\
\bar{\kappa}_{2} \sigma_{6}+\tilde{\sigma}_{5} & -\bar{\kappa}_{2} \gamma_{1}-\tilde{\sigma}_{5}-\bar{\kappa}_{2} \tilde{\sigma}_{6} & 0 & 0
\end{array}\right] .
$$

Since $\mathbf{P}_{x}$ is positive definite and the Kronecker product preserves positive definiteness, the necessary requirement for (50) to be bounded is that the penalty coefficients are chosen such that $M \geq 0$. The penalty coefficients are given in

Theorem 1. The coupling terms $I T_{3}$ in (50) are bounded using

$$
\tilde{\sigma}_{4}=\sigma_{4} \leq 0, \quad \sigma_{5}=-\bar{\kappa}_{1} r, \quad \sigma_{6}=\gamma+r, \quad \tilde{\sigma}_{5}=-\bar{\kappa}_{2}\left(\gamma_{1}+r\right), \quad \tilde{\sigma}_{6}=r, \quad r \in \mathbb{R}
$$


and hence the scheme (41) is stable.

Proof. With the choices of penalty coefficients given in Proposition 1, the matrix $M$ in (53) reduces to

$$
M=2 \sigma_{4}\left[\begin{array}{cccc}
-1 & 1 & 0 & 0 \\
1 & -1 & 0 & 0 \\
0 & 0 & 0 & 0 \\
0 & 0 & 0 & 0
\end{array}\right]
$$

with eigenvalues $\lambda_{1,2,3}=0$ and $\lambda_{4}=-4 \sigma_{4}$. Hence if $\sigma_{4} \leq 0$ we have $M \geq 0$.

\section{Numerical results}

To verify the numerical scheme we use what is often called the method of manufactured solutions $[4,31]$. We chose the solution and use that to compute a righthand-side forcing function, initial- and boundary data. According to the principle of Duhamel [32], the number or form of the boundary conditions does not change due to the addition of the forcing function. We can hence test the convergence of the scheme towards this analytical solution. The interface conditions (32) are of course not satisfied in general by this solution and we need to modify them by adding a right-hand-side. 
We use the manufactured solution

$$
\begin{aligned}
& \rho(x, y, t)=1+\eta \sin (\theta \pi(x-y)-t)^{2} \\
& u(x, y, t)=\eta \cos (\theta \pi(x+y)-t) \\
& v(x, y, t)=\eta \sin (\theta \pi(x-y)-t) \\
& p(x, y, t)=1+\eta \cos (\theta \pi(x+y)-t)^{2},
\end{aligned}
$$

with different values of $\eta, \theta$ in the fluid and solid domains, to generate the solution. The energy and temperature can be computed using (11) and (12). Since the stability of the scheme is independent of the order of accuracy, the difference operators is the only thing which have to be changed in order to achieve higher, or lower, accuracy. The rate of convergence, $Q$, is computed as

$$
Q^{(j)}=\frac{1}{\log \left(\frac{N_{i+1}}{N_{i}}\right)} \log \left(\frac{E_{i}^{(j)}}{E_{i+1}^{(j)}}\right)
$$

for each of the conserved varables $q^{(j)}, j=1,2,3,4$. We have used the same number of grid points, $N$, in both coordinate directions for both the fluid and solid domain. $N_{k}$ denotes the number of gridpoints at refinement level $k$ and $E_{k}^{(j)}$ is the $L_{2}$-error between the computed and exact solution for each conserved variable. The time integration is done with the classical 4th-order Runge-Kutta method until time $t=$ 0.1 using 1000 time steps.

In Table A.1 we list the convergence results for the conserved variables for both the fluid and solid domains. As we can see from Table A.1 we can achieve 5th-order accuracy by simply replacing the difference operators. No other modifications to the 
scheme is necessary.

[Table 1 about here.]

6.1. Comparison of the different approaches to the conjugate heat transfer problem

When the heat transfer in the solid is governed by the compressible Navier-Stokes equations, one does not solve the same conjugate heat transfer problem as when the heat transfer is governed by the heat equation. This is because the relations in (21) holds only approximately as time passes. The exchange of heat between the fluid and solid domains will affect the temperature and hence also the density, because of the equation of state, and introduce small density variations in the solid domain. We can numerically solve the conjugate heat transfer problem in both ways and determine the difference between the two methods. Note that we do not overwrite, or enforce, the velocities to zero inside the solid domain. The velocities are weakly enforced to zero at the boundaries and interfaces only.

Let NS-NS denote the case when the heat transfer is governed by the compressible Navier-Stokes equations and NS-HT the case where the heat transfer is governed by the heat equation. The well-posedness and stability of NS-HT coupling is proven in the appendix. The initial and boundary data are chosen such that NS-NS and NSHT have identical solutions initially, and we study the differences of the two methods over time.

To quantify the difference between the two methods, NS-NS and NS-HT, we compute two representative cases. The computational domain is $\Omega=\Omega_{1} \cup \Omega_{2}$ where $\Omega_{1}=[0,1] \times[0,1]$ and $\Omega_{2}=[0,1] \times[-1,0]$. All computations are done using 3rd-order 
accurate SBP operators and the time integration is done using the classical 4th-order Runge-Kutta method.

In the first case, the computations are initialized with zero velocities everywhere and temperature $T=1$ in both subdomains. In the $x$-direction we have chosen periodic boundary conditions. At $y=-1$ we specify $T=1.5$ and at $y=1$ we have $T=1$. For the Navier-Stokes equations we have no-slip solid walls as described in [26] for the velocities. These choices of boundary conditions renders the solution to be homogeneous in the $x$-direction.

Under the assumption of identically zero velocities and periodicity in the $x$ direction, the exact steady-state solution can be obtained as

$$
\begin{aligned}
& T=-\frac{k}{2(k+1)} y+\frac{3 k+2}{2(k+1)}, \\
& \tilde{T}=-\frac{1}{2(k+1)} y+\frac{3 k+2}{2(k+1)},
\end{aligned}
$$

where $k=\kappa_{2} / \kappa_{1}$ is the ratio of the steady-state thermal conductivities. We can see from (58) that the only occurring material parameter is the ratio between the thermal conductivity coefficients. Neither the density nor the thermal diffusivity has any effect on the steady-state solution. The larger the ratio of the thermal conductivities is, the stiffer the problem becomes. In the calculations below, we have chosen the parameters such that $k=5$.

The temperature distribution at time $t=500$, which is the steady-state solution, is seen in Figure A.1 when using 65 grid points in each coordinate direction and subdomain. In Figure A.2 we show an intersection of the absolute difference along 
the line $-1 \leq y \leq 1$ at $x=0.5$ together with the time-evolution of the $l_{\infty^{-}}$and $l_{2}$-differences. In Figure $2(\mathrm{~b})$ we can see that the large initial discontinuity gives differences in the beginning of the computation. As the velocities are damped over time, the difference decreases rapidly towards zero.

[Figure 1 about here.]

[Figure 2 about here.]

In Table A.2 we list the results for different number of grid points.

[Table 2 about here.]

As we can see from Table A.2, the differences are very small. Even for the coarsest mesh, the relative maximum and interface differences are less than $0.1 \%$ while the relative $l_{2}$-difference is approximately $0.05 \%$. Note that the differences are decreasing with the resolution. The steady-state solutions will become identical as the mesh is further refined.

Next, we consider an unsteady problem. The boundary data at the south boundary is perturbed by the time-dependent perturbation

$$
f(x, t)=1+0.25 * \sin (t) * \sin (\pi x)
$$

and hence there will be no steady-state solution. In the $x$-direction in the solid domain, we have changed from periodic boundary conditions to solid wall boundary conditions with prescribed temperature $T=1$. This is a more realistic way to enclose 
the solid domain, and it has the additional benefit of damping the induced velocities in the Navier-Stokes equations.

The results can be seen in Figure (A.3). We plot the $l_{\infty^{-}}$and $l_{2}$-difference as a function of time. As we can see, the difference does not approach zero but remains bounded and small. The relative mean difference is less than $0.5 \%$ while the maximum difference is less than $1.5 \%$. Thus, despite the rather large variation in the boundary data, NS-NS and NS-HT produces very similar solutions.

[Figure 3 about here.]

In a CFD computation, the part of the domain which is solid is in general small compared to the fluid domain, for example when computing the flow field around an airfoil or aircraft. Despite the Navier-Stokes equations being significantly more expensive to solve, the overall additional cost of solving the Navier-Stokes equations also in the solid is in general limited.

\subsection{A numerical example of conjugate heat transfer}

As a final computational example, we consider the coupling of a flow over a slab of material for which the ratio of the thermal conductivities is 100 . The initial temperature condition is $T=1$ in the fluid domain and $\tilde{T}=1.5$ in the solid domain. The boundary conditions are periodic in the $x$-direction. At the south boundary, $y=-1$, in the solid domain we let $\tilde{T}=1.5$ and at the north boundary, $y=1$, in the fluid domain, there is a Mach 0.5 free-stream boundary condition with $T=1$, as described in [28]. Figure A.4 shows a snapshot of the solution at time $t=2.5$. The 
velocity components in the solid domain are zero to machine precision and the heat transfer in the solid is exclusively driven by diffusion.

[Figure 4 about here.]

\section{Conclusions}

We have proven that a conjugate heat transfer coupling of the compressible Navier-Stokes equations is well-posed when a modified norm is used. The equations were discretized using a finite difference method on summation-by-parts form with boundary- and interface conditions imposed weakly by the simultaneous approximation term. It was shown that a modified discrete norm was needed in order to prove energy stability of the scheme. The stability is independent of the order of accuracy, and it was shown that we can achieve all orders of accuracy by simply using higher order accurate SBP operators.

We showed that the difference when the heat transfer is governed by the heat equation, compared to the compressible Navier-Stokes equations, is small. The steady-state solutions differed by less than $0.005 \%$ as the mesh was refined while a perturbed, unsteady solution differed by less than $0.5 \%$ on average.

There are many multi-block codes for the compressible Navier-Stokes equations available. To implement conjugate heat transfer is significantly easier with the method of modifying the interface conditions, rather than coupling to a different physics solver for the heat transfer part. While the Navier-Stokes equations are more expensive to solve, usually only a small part of the computational domain is solid and the heat transfer is computed at a low additional cost. 


\section{Acknowledgments}

The computations were performed on resources provided by SNIC through Uppsala Multidisciplinary Center for Advanced Computational Science (UPPMAX) under Project p2010056.

\section{Appendix A. Coupling of the compressible Navier-Stokes equations with the heat equation}

In [4], a model problem for conjugate heat transfer was considered. The equations

were one-dimensional, linear and symmetric. In this appendix we extend the work to the two-dimensional compressible, non-linear Navier-Stokes equations coupled with the heat equation in two space dimensions. The well-posedness of the coupling is shown in

Proposition 2. The compressible Navier-Stokes equations coupled with the heat equation, is well-posed with the interface conditions

$$
T=\tilde{T}, \kappa T_{y}=\kappa_{s} \tilde{T}_{y}
$$

for the temperature, and the no-slip ${ }^{1}$ conditions

$$
u=0, \quad v=0
$$

for the velocities.

\footnotetext{
${ }^{1}$ See [26] for more general conditions.
} 
Proof. Consider the heat equation (15) and the Navier-Stokes equations in the constant, linear, symmetric formulation. The estimates of $w$ and $\tilde{T}$ will be derived in the $L^{2}$-equivalent norms

$$
\|w\|_{J_{1}}^{2}=\int_{\Omega_{1}} w^{T} J_{1} w d \Omega_{1}, \quad\|\tilde{T}\|_{\nu_{2}}^{2}=\int_{\Omega_{2}} \tilde{T}^{2} \nu_{2} d \Omega_{2}
$$

where $J_{1}=\operatorname{diag}\left[1,1,1, \nu_{1}\right]$ and $\nu_{1,2}>0$ are to be determined.

Remember that the symmetrized variables for the Navier-Stokes equations are

$$
w=\left[\frac{\bar{c}}{\sqrt{\gamma} \bar{\rho}} \rho, u, v, \frac{1}{\bar{c} \sqrt{\gamma(\gamma-1)}} T\right]^{T} .
$$

and note that there is a scaling coefficient in the temperature component. To simplify the analysis, we rescale (15) by multiplying the equation with $\frac{1}{\bar{c} \sqrt{\gamma(\gamma-1}}$. To apply the energy method, we rewrite the speed of sound based Péclet number $P e_{c}$ in (14) as

$$
P e_{c}=\frac{P r \cdot R e}{M a}=\frac{P r}{\varepsilon}
$$

where $\operatorname{Pr}$ is the Prandtl number. Then (15) becomes

$$
\frac{\tilde{T}_{t}}{\bar{c} \sqrt{\gamma(\gamma-1)}}=\frac{\varepsilon \kappa_{s}}{\operatorname{Pr} \bar{c} \sqrt{\gamma(\gamma-1)} \rho_{s} c_{s}}\left(\tilde{T}_{x x}+\tilde{T}_{y y}\right)
$$


By applying the energy method to each equation and adding the results we obtain

$$
\frac{d}{d t}\left(\|w\|_{J_{1}}^{2}+\frac{1}{\bar{c}^{2} \gamma(\gamma-1)}\|\tilde{T}\|_{\nu_{2}}^{2}\right) \leq \frac{-2 \varepsilon}{\bar{c}^{2} \gamma(\gamma-1) P r} \int_{0}^{1}\left(\frac{\nu_{1} \gamma \mu}{\bar{\rho}} T T_{y}-\frac{\nu_{2} \kappa_{s}}{\rho_{s} c_{s}} \tilde{T} \tilde{T}_{y}\right) d x
$$

If we choose

$$
\nu_{1}=\frac{\bar{\kappa} \bar{\rho}}{\gamma \mu}, \quad \nu_{2}=\rho_{s} c_{s}
$$

and apply the interface conditions (A.1) we get

$$
\frac{d}{d t}\left(\|w\|_{J_{1}}^{2}+\frac{1}{\bar{c}^{2} \gamma(\gamma-1)}\|\tilde{T}\|_{\nu_{2}}^{2}\right) \leq \frac{-2 \varepsilon}{\bar{c}^{2} \gamma(\gamma-1) P r} \int_{0}^{1} T\left(\bar{\kappa} T_{y}-\kappa_{s} \tilde{T}_{y}\right) d x=0
$$

and hence the conditions (A.1) does not contribute to unbounded energy growth.

Note again that the application of the physical interface conditions (A.1) requires the use of a non-standard norm in the energy estimates. All quantities involved in the weights $\nu_{1,2}$ are, however, always positive and they will hence always define a norm.

The discretization of the coupled system is analogous to that which is presented in [4], and extended to multiple dimensions as described before. We hence only present the numerical scheme and the choice of interface penalty coefficients such that the scheme is stable.

An SBP-SAT discretization of the Navier-Stokes equations coupled with the heat 
equation is given by, when only considering the interface terms,

$$
\begin{aligned}
\mathbf{w}_{t}+\left(\bar{D}_{x} \otimes I_{4}\right) \mathbf{F}+\left(\bar{D}_{y} \otimes I_{4}\right) \mathbf{G} & =\mathbb{S} \\
\tilde{\mathbf{T}}_{t}-\left(\bar{D}_{x}^{2} \tilde{\mathbf{T}}+\bar{D}_{y}^{2} \tilde{\mathbf{T}}\right) & =\tilde{\mathbb{S}}
\end{aligned}
$$

The penalty terms are given by

$$
\begin{aligned}
\mathbb{S} & =\left(\bar{P}_{y}^{-1} \bar{E}_{x, y_{N}} \otimes \bar{\Sigma}_{1}\right)\left(\mathbf{w}-g^{I}\right) \\
& +\varepsilon \sigma_{2}\left(\bar{P}_{y}^{-1} \bar{E}_{x, y_{N}} \otimes I_{4}\right)\left(\bar{H}_{2} \mathbf{w}-g_{1}\right) \\
& +\varepsilon \sigma_{3}\left(\bar{P}_{y}^{-1} \bar{E}_{x, y_{N}} \otimes I_{4}\right)\left(\bar{H}_{3} \mathbf{w}-g_{2}\right) \\
& +\varepsilon\left(\bar{P}_{y}^{-1} \bar{E}_{x, y_{N}} \otimes I_{4}\right) \bar{\Theta}_{1}\left(\bar{H}_{3}\left(\bar{D}_{x} \otimes I_{4}\right) \mathbf{w}-\frac{\partial g_{2}}{\partial x}\right) \\
& +\varepsilon\left(\bar{P}_{y}^{-1} \bar{E}_{x, y_{N}} \otimes \Sigma_{4}\right)\left(\bar{I}_{1}^{T} \mathbf{w}-\bar{I}_{2}^{T}\left(\tilde{\mathbf{T}} \otimes \mathbf{e}_{\mathbf{4}}\right)\right) \\
& +\varepsilon\left(\bar{P}_{y}^{-1} \bar{D}_{y}^{T} \bar{E}_{x, y_{N}} \otimes \Sigma_{5}\right)\left(\bar{I}_{1}^{T} \mathbf{w}-\bar{I}_{2}^{T}\left(\tilde{\mathbf{T}} \otimes \mathbf{e}_{\mathbf{4}}\right)\right) \\
& +\varepsilon\left(\bar{P}_{y}^{-1} \bar{E}_{x, y_{N}} \otimes \Sigma_{6}\right)\left(\bar{\kappa} \bar{I}_{1}^{T}\left(\bar{D}_{y} \otimes I_{4}\right) \mathbf{w}-\kappa_{s} \bar{I}_{2}^{T}\left(\bar{D}_{y} \tilde{\mathbf{T}} \otimes \mathbf{e}_{\mathbf{4}}\right)\right)
\end{aligned}
$$

where $\Sigma_{4,5,6}=\operatorname{diag}\left[0,0,0, \sigma_{4,5,6}\right]$ and the term involving $\bar{\Theta}_{1}$ is explained in Remark 3 . The SAT for the heat equation is given by

$$
\begin{aligned}
\tilde{\mathbb{S}} & =\varepsilon \tau_{4} \bar{P}_{y}^{-1} \bar{E}_{x, y_{N}}(\tilde{\mathbf{T}}-\mathbf{T})+\varepsilon \tau_{5} \bar{P}_{y}^{-1} \bar{D}_{y}^{T} \bar{E}_{x, y_{N}}(\tilde{\mathbf{T}}-\mathbf{T}) \\
& +\varepsilon \tau_{6} \bar{P}_{y}^{-1} \bar{E}_{x, y_{N}}\left(\kappa_{s} \bar{D}_{y} \tilde{\mathbf{T}}-\bar{\kappa} \bar{D}_{y} \mathbf{T}\right)
\end{aligned}
$$

and the choices of penalty parameters such that the coupled scheme is stable is given in

Theorem 2. The scheme (A.10) for coupling the Navier-Stokes equations with the 
heat equation is stable with the SATs given by (A.11), (A.12) where the penalty coefficients for the coupling terms are given by

$$
\begin{aligned}
& r \in \mathbb{R}, \\
& \sigma_{4}=\tau_{4} \leq 0, \quad \sigma_{5}=-\kappa_{s} r, \quad \sigma_{6}=\frac{-1+r P r}{\operatorname{Pr}}, \quad \tau_{5}=-\frac{\bar{\kappa}(-1+r \operatorname{Pr})}{\operatorname{Pr}}, \quad \tau_{6}=r .
\end{aligned}
$$

Proof. We apply the energy method, using the modified discrete norms,

$$
\|\mathbf{w}\|_{J_{1}}^{2}=\mathbf{w}^{T}\left(\bar{P} \otimes J_{1}\right) w, \quad\|\tilde{\mathbf{T}}\|_{\nu_{2}}^{2}=\nu_{2} \tilde{\mathbf{T}}^{T} \bar{P} \mathbf{T}
$$

where $J_{1}=\operatorname{diag}\left[1,1,1, \nu_{1}\right]$ and $\nu_{1,2}$ are given in (A.8). Using appropriate penalty terms for the inviscid part and the velocity components of the Navier-Stokes equation, see $[33,26]$, we obtain the energy estimate

$$
\frac{d}{d t}\|\mathbf{w}\|_{J_{1}}^{2}+\frac{d}{d t}\|\tilde{\mathbf{T}}\|_{\nu_{2}}^{2} \leq 0
$$

when using the penalty coefficients given in (A.13).

\section{References}

[1] M. B. Giles. Stability analysis of numerical interface conditions in fluidstructure thermal analysis. International Journal for Numerical Methods in Fluids, 25:421-436, August 1997.

[2] B. Roe, R. Jaiman, A. Haselbacher, and P. H. Geubelle. Combined inter- 
face boundary condition method for coupled thermal simulations. International Journal for Numerical Methods in Fluids, 57:329-354, May 2008.

[3] William D. Henshaw and Kyle K. Chand. A composite grid solver for conjugate heat transfer in fluid-structure systems. Journal of Computational Physics, 228(10):3708-3741, 2009.

[4] Jens Lindström and Jan Nordström. A stable and high-order accurate conjugate heat transfer problem. Journal of Computational Physics, 229(14):5440-5456, 2010.

[5] Michael Schäfer and Ilka Teschauer. Numerical simulation of coupled fluidsolid problems. Computer Methods in Applied Mechanics and Engineering, 190(28):3645-3667, 2001.

[6] Niphon Wansophark, Atipong Malatip, and Pramote Dechaumphai. Streamline upwind finite element method for conjugate heat transfer problems. Acta Mechanica Sinica, 21:436-443, 2005.

[7] É. Turgeon, D. Pelletier, and F. Ilinca. Compressible heat transfer computations by an adaptive finite element method. International Journal of Thermal Sciences, 41(8):721-736, 2002.

[8] Xi Chen and Peng Han. A note on the solution of conjugate heat transfer problems using simple-like algorithms. International Journal of Heat and Fluid Flow, 21(4):463-467, 2000. 
[9] Magnus Svärd, Ken Mattsson, and Jan Nordström. Steady-state computations using summation-by-parts operators. Journal of Scientific Computing, 24(1):7995, 2005.

[10] Ken Mattsson, Magnus Svärd, Mark Carpenter, and Jan Nordström. Highorder accurate computations for unsteady aerodynamics. Computers and Fluids, $36(3): 636-649,2007$.

[11] X. Huan, Jason E. Hicken, and David W. Zingg. Interface and boundary schemes for high-order methods. In the 39th AIAA Fluid Dynamics Conference, AIAA Paper No. 2009-3658, San Antonio, USA, 22-25 June 2009.

[12] Jan Nordström, Jing Gong, Edwin van der Weide, and Magnus Svärd. A stable and conservative high order multi-block method for the compressible NavierStokes equations. Journal of Computational Physics, 228(24):9020-9035, 2009.

[13] Ken Mattsson, Magnus Svärd, and Mohammad Shoeybi. Stable and accurate schemes for the compressible Navier-Stokes equations. Journal of Computational Physics, 227:2293-2316, February 2008.

[14] Jan Nordström, Sofia Eriksson, Craig Law, and Jing Gong. Shock and vortex calculations using a very high order accurate Euler and Navier-Stokes solver. Journal of Mechanics and MEMS, 1(1):19-26, 2009.

[15] Jan Nordström, Frank Ham, Mohammad Shoeybi, Edwin van der Weide, Magnus Svärd, Ken Mattsson, Gianluca Iaccarino, and Jing Gong. A hybrid method for unsteady inviscid fluid flow. Computers \& Fluids, 38:875-882, 2009. 
[16] Heinz-Otto Kreiss and Godela Scherer. Finite element and finite difference methods for hyperbolic partial differential equations. In Mathematical Aspects of Finite Elements in Partial Differential Equations, number 33 in Publ. Math. Res. Center Univ. Wisconsin, pages 195-212. Academic Press, 1974.

[17] Heinz-Otto Kreiss and Godela Scherer. On the existence of energy estimates for difference approximations for hyperbolic systems. Technical report, Uppsala University, Division of Scientific Computing, 1977.

[18] Mark H. Carpenter, David Gottlieb, and Saul Abarbanel. Time-stable boundary conditions for finite-difference schemes solving hyperbolic systems: Methodology and application to high-order compact schemes. Journal of Computational Physics, 111(2):220-236, 1994.

[19] Mark H. Carpenter, Jan Nordström, and David Gottlieb. A stable and conservative interface treatment of arbitrary spatial accuracy. Journal of Computational Physics, 148(2):341-365, 1999.

[20] Jan Nordström and Magnus Svärd. Well-posed boundary conditions for the Navier-Stokes equations. SIAM Journal on Numerical Analysis, 43(3):12311255, 2005.

[21] Bo Strand. Summation by parts for finite difference approximations for $d / d x$. Journal of Computational Physics, 110(1):47-67, 1994.

[22] Ken Mattsson and Jan Nordström. Summation by parts operators for finite dif- 
ference approximations of second derivatives. Journal of Computational Physics, 199(2):503-540, 2004.

[23] Ken Mattsson. Summation by parts operators for finite difference approximations of second-derivatives with variable coefficients. Journal of Scientific Computing, pages 1-33, 2011.

[24] Jorg U. Schlüter, Xiaohua Wu, Edwin van der Weide, S. Hahn, Juan J. Alonso, and Heinz Pitsch. Multi-code simulations: A generalized coupling approach. In the 17th AIAA CFD Conference, AIAA-2005-4997, Toronto, Canada, June 2005.

[25] Magnus Svärd and Jan Nordström. On the order of accuracy for difference approximations of initial-boundary value problems. Journal of Computational Physics, 218(1):333-352, 2006.

[26] Jens Berg and Jan Nordström. Stable Robin solid wall boundary conditions for the Navier-Stokes equations. Journal of Computational Physics, 230(19):7519$7532,2011$.

[27] Saul Abarbanel and David Gottlieb. Optimal time splitting for two- and threedimensional Navier-Stokes equations with mixed derivatives. Journal of Computational Physics, 41(1):1-33, 1981.

[28] Magnus Svärd, Mark H. Carpenter, and Jan Nordström. A stable high-order finite difference scheme for the compressible Navier-Stokes equations, far-field 
boundary conditions. Journal of Computational Physics, 225(1):1020-1038, 2007.

[29] Heinz-Otto Kreiss and Jens Lorenz. Initial-Boundary Value Problems and the Navier-Stokes Equations. Classics in Applied Mathematics. Society for Industrial and Applied Mathematics, 2004.

[30] Ken Mattsson and Mark H. Carpenter. Stable and accurate interpolation operators for high-order multiblock finite difference methods. SIAM Journal on Scientific Computing, 32(4):2298-2320, 2010.

[31] Lee Shunn, Frank Ham, and Parviz Moin. Verification of variable-density flow solvers using manufactured solutions. Journal of Computational Physics, 231(9):3801-3827, 2012.

[32] Bertil Gustafsson, Heinz-Otto Kreiss, and Joseph Oliger. Time Dependent Problems and Difference Methods. Wiley Interscience, 1995.

[33] Magnus Svärd and Jan Nordström. A stable high-order finite difference scheme for the compressible Navier-Stokes equations: No-slip wall boundary conditions. Journal of Computational Physics, 227(10):4805-4824, 2008. 


\section{List of Figures}

A.1 Temperatures at time $t=500$ from NS-NS and NS-HT using 65 grid points in each coordinate direction and subdomain . . . . . . . . . . . 40

A.2 Temperature intersection and time differences for NS-NS and NS-HT using 65 grid points in each coordinate direction and subdomain . . . 41

A.3 $l_{\infty^{-}}$and $l_{2}$-difference in time between NS-HS and NS-HT for an unsteady problem . . . . . . . . . . . . . . . . 42

A.4 Temperature and velocity profiles for a flow past a slab of material using 65x65 grid points in both domains . . . . . . . . . . . . 43 


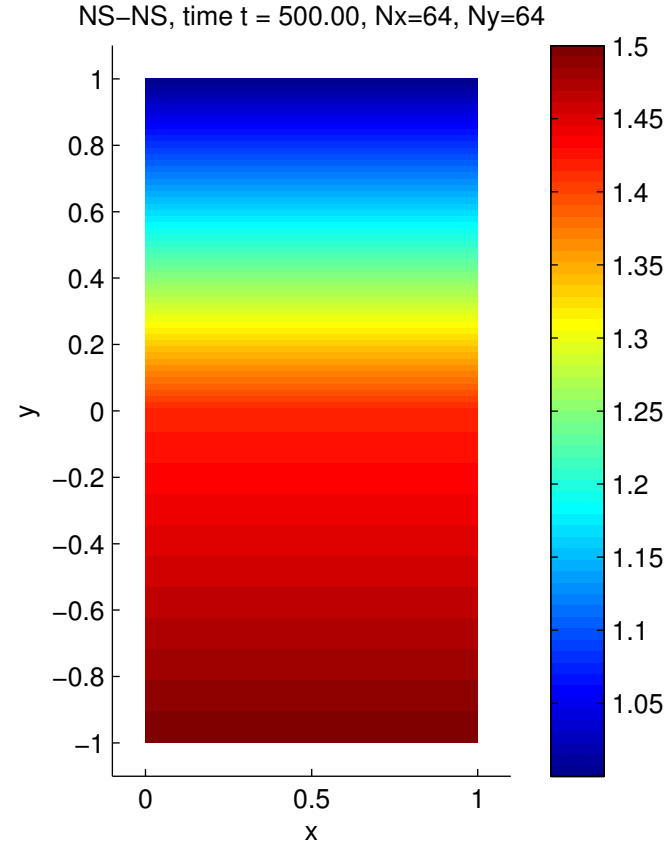

(a) Temperature distribution from NS-NS

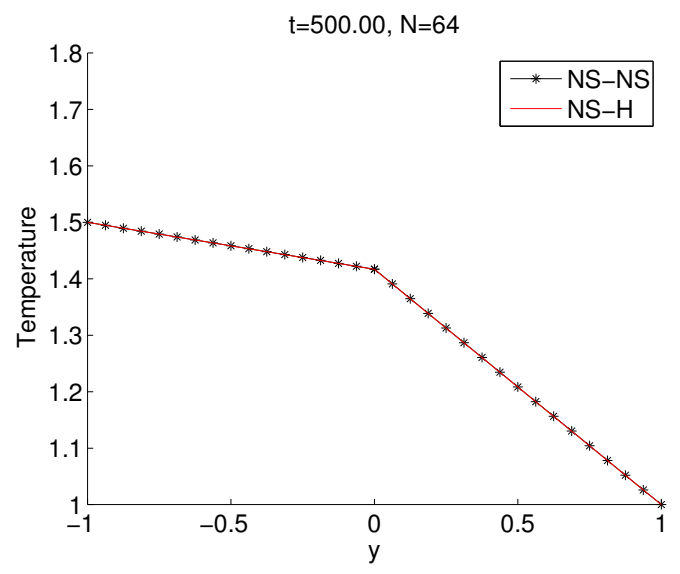

(b) Intersection along $y$ at $x=0.5$ of the temperature distribution for NS-NS and NS-HT

Figure A.1: Temperatures at time $t=500$ from NS-NS and NS-HT using 65 grid points in each coordinate direction and subdomain 


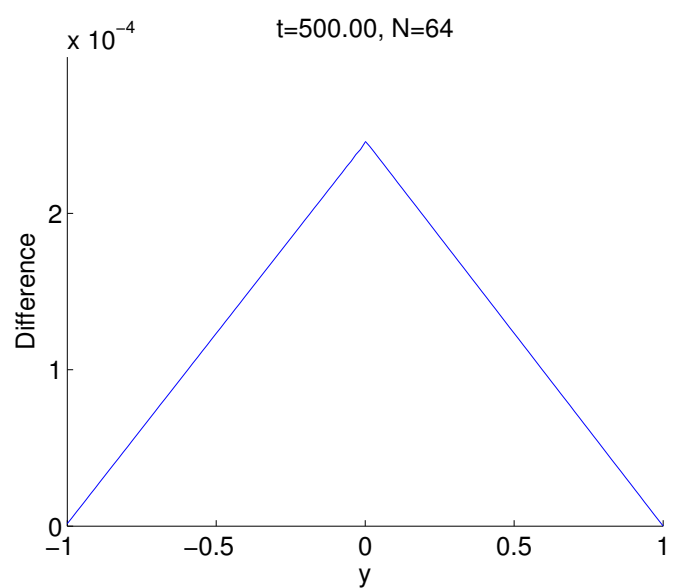

(a) Intersection along $y$ at $x=0.5$ of the absolute difference in temperature distribution between NS-NS and NS-HT

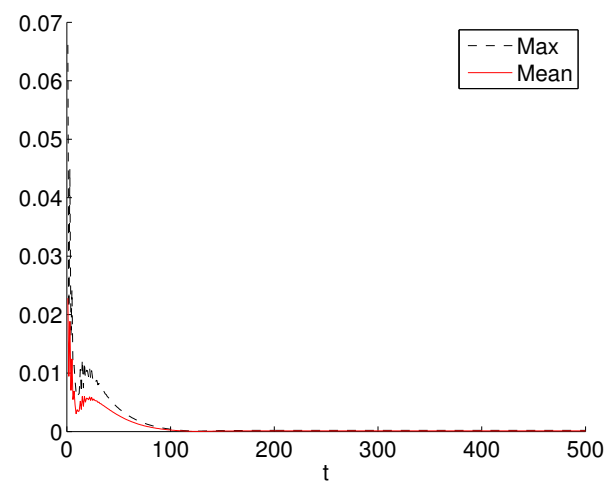

(b) $l_{\infty}$ - and $l_{2}$-difference in time

Figure A.2: Temperature intersection and time differences for NS-NS and NS-HT using 65 grid points in each coordinate direction and subdomain 


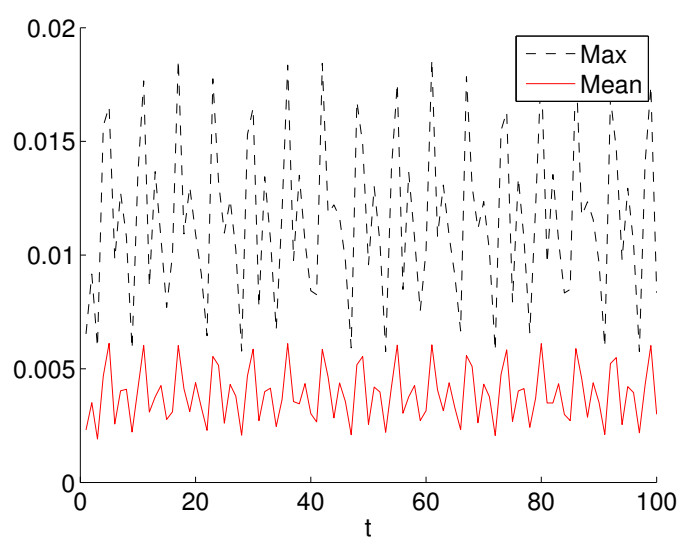

Figure A.3: $l_{\infty}$ - and $l_{2}$-difference in time between NS-HS and NS-HT for an unsteady problem 


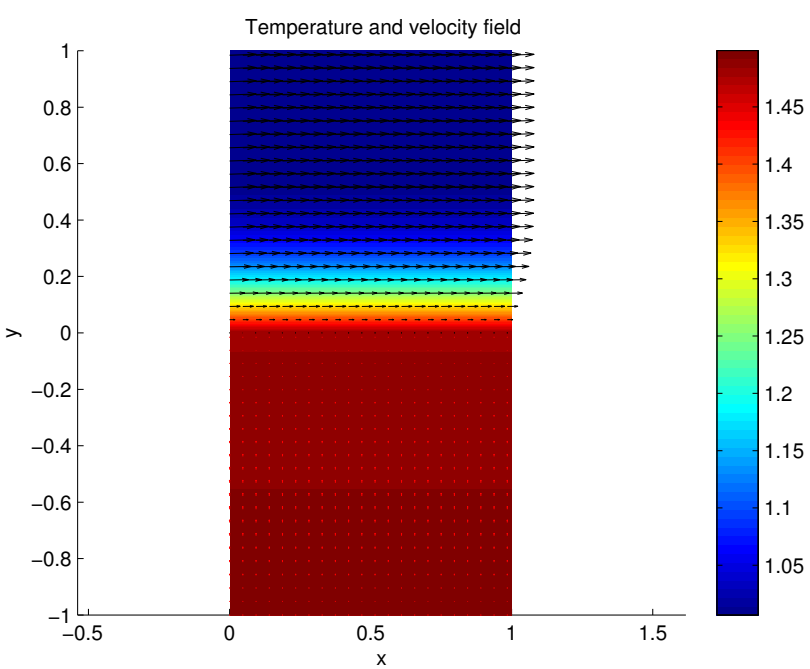

(a) Temperature distribution and velocity profile

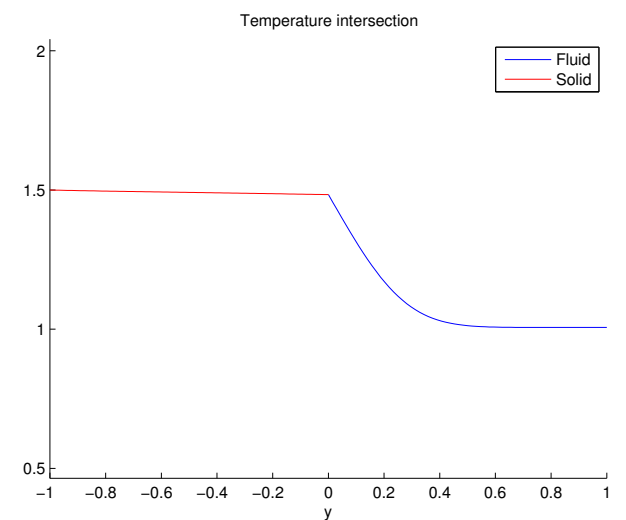

(b) Intersection of the temperature distribution along $x=0.5$.

Figure A.4: Temperature and velocity profiles for a flow past a slab of material using $65 \times 65$ grid points in both domains 


\section{List of Tables}

A.1 Convergence results for the conjugate heat transfer problem . . . . . 45

A.2 Difference between NS-NS and NS-HT at time $t=500 \ldots$. . . . . . 46 
Table A.1: Convergence results for the conjugate heat transfer problem

\begin{tabular}{|c|c|c|c||c|c|c|}
\hline & \multicolumn{3}{|c||}{ 2nd-order } & \multicolumn{3}{c|}{ 3rd-order } \\
\hline$N$ & $32 / 64$ & $64 / 96$ & $96 / 128$ & $32 / 64$ & $64 / 96$ & $96 / 128$ \\
\hline$\rho$ & 1.8367 & 1.8931 & 2.0133 & 2.6222 & 3.0699 & 3.4795 \\
$\rho u$ & 2.0824 & 2.0803 & 2.1187 & 2.9846 & 3.0748 & 3.1927 \\
$\rho v$ & 2.0503 & 2.0549 & 2.0922 & 3.4222 & 3.7512 & 3.4199 \\
$e$ & 1.8174 & 1.9065 & 1.9963 & 2.4639 & 2.7749 & 3.0523 \\
\hline$\tilde{\rho}$ & 1.8933 & 1.8533 & 1.9628 & 2.5761 & 2.9791 & 3.5767 \\
$\tilde{\rho} \tilde{u}$ & 2.0544 & 2.0803 & 2.0992 & 3.1094 & 3.0374 & 3.2732 \\
$\tilde{\rho} \tilde{v}$ & 1.9411 & 2.0190 & 2.0894 & 3.3928 & 3.7465 & 3.3628 \\
$\tilde{e}$ & 1.9483 & 1.9151 & 1.9409 & 2.9451 & 2.8399 & 3.2560 \\
\hline \hline & \multicolumn{3}{|c||}{4 th-order } & \multicolumn{3}{|c||}{5 th-order } \\
\hline$N$ & $32 / 64$ & $64 / 96$ & $96 / 128$ & $32 / 64$ & $64 / 96$ & $96 / 128$ \\
\hline$\rho$ & 3.9662 & 4.1381 & 4.1138 & 4.4824 & 5.2584 & 5.5131 \\
$\rho u$ & 4.4531 & 4.3640 & 4.2799 & 4.6819 & 4.7521 & 4.6733 \\
$\rho v$ & 4.3175 & 4.0918 & 4.0284 & 4.9824 & 4.9257 & 4.7839 \\
$e$ & 3.9757 & 4.1723 & 4.0957 & 4.3760 & 4.6227 & 4.7207 \\
\hline$\tilde{\rho}$ & 3.9935 & 4.3902 & 4.5538 & 4.4421 & 5.1497 & 5.5388 \\
$\tilde{\rho} \tilde{u}$ & 4.2072 & 4.3159 & 4.4366 & 4.9665 & 4.9739 & 4.9512 \\
$\tilde{\rho} \tilde{v}$ & 4.3672 & 4.3331 & 4.3212 & 5.1007 & 5.1370 & 4.9087 \\
$\tilde{e}$ & 3.9025 & 4.3178 & 4.4091 & 4.8746 & 4.8573 & 4.9518 \\
\hline
\end{tabular}


Table A.2: Difference between NS-NS and NS-HT at time $t=500$

\begin{tabular}{|c|c|c|c|}
\hline & \multicolumn{3}{|c|}{ Difference } \\
\hline$N$ & $l_{\infty}$ & $l_{2}$ & Interface \\
\hline 32 & $1.1514 \mathrm{e}-03$ & $6.8992 \mathrm{e}-04$ & $1.1514 \mathrm{e}-03$ \\
64 & $2.4612 \mathrm{e}-04$ & $1.4491 \mathrm{e}-04$ & $2.4612 \mathrm{e}-04$ \\
128 & $4.3440 \mathrm{e}-05$ & $2.5329 \mathrm{e}-05$ & $4.3440 \mathrm{e}-05$ \\
\hline
\end{tabular}

